\title{
AVERAGING OF EARTH-CROSSING ORBITS
}

\author{
G.F. GRONCHI and A. MILANI \\ Department of Mathematics, University of Pisa
}

The orbits of planet-crossing asteroids (and comets) can undergo close approaches and collisions with some major planet. This introduces a singularity in the $\mathrm{N}$-body Hamiltonian, and the averaging of the equations of motion, traditionally used to compute secular perturbations, is undefined. We have shown (Gronchi and Milani, 1998) that it is possible to define in a rigorous way some generalised averaged equations of motion, in such a way that the generalised solutions are unique and piecewise smooth, with comers on the node crossing lines.

The model is the averaged equations of motion first introduced by Kozai (1962): the perturbing planets are assumed to move in circular, coplanar orbits, and the equations of motion are averaged over the anomalies of the asteroid and of the planets. In the non-crossing case the averaging is integrable; in the planet-crossing case there is a polar singularity of order two in the equations of motion, and

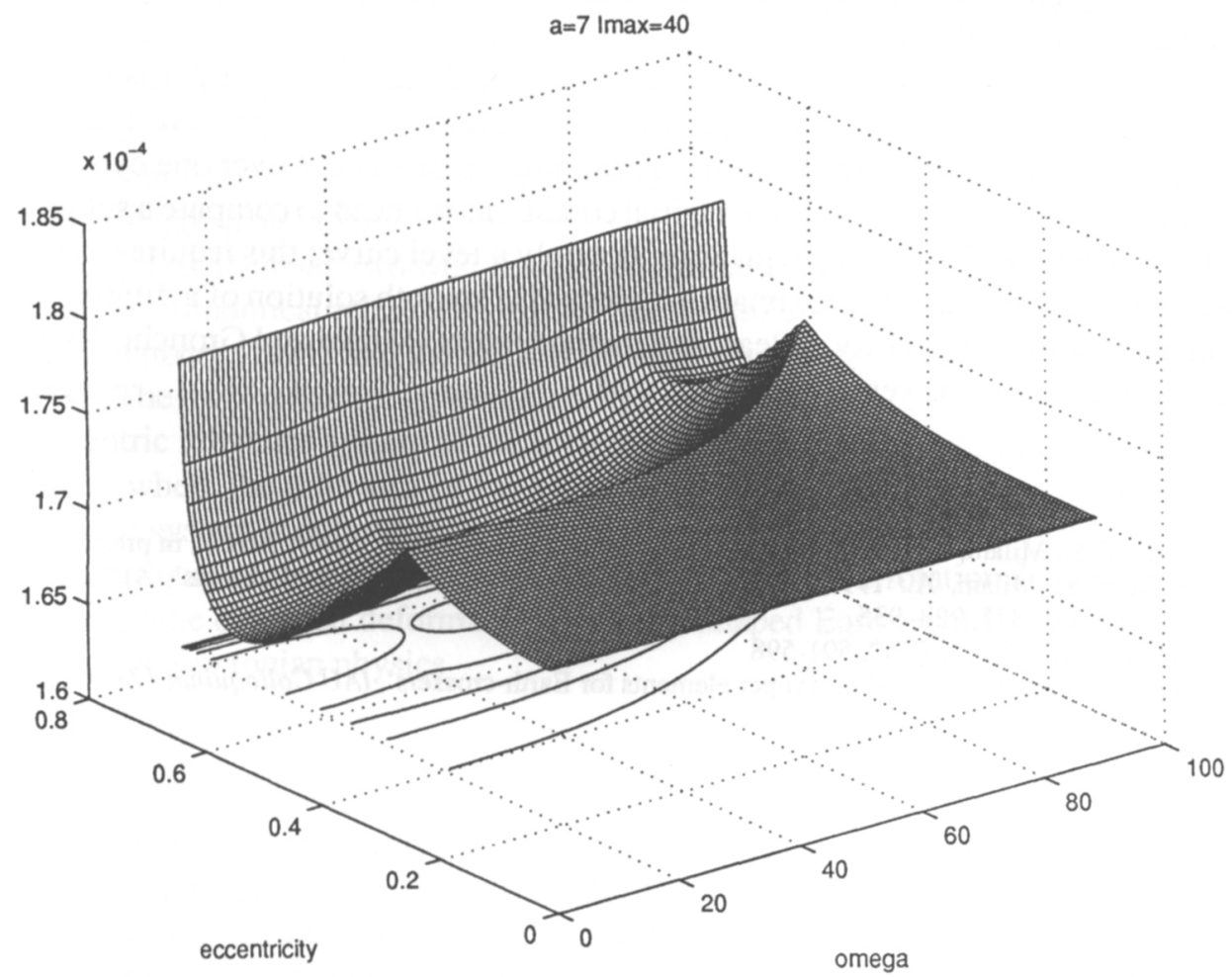

Fig. 1. Graph of the averaged perturbing function for a Centaur type orbit. The node crossing lines with Jupiter appear as crests, the double crossing point as a pyramid-like maximum, and the stable Kozai librations as minima. 
averaging is not possible. To define a generalized solution, we decrease the order of the polar singularity by the method of extraction of the singularities by Kantorovich. The singularity of the perturbing function is approximated by a modified inverse distance, the one between the straight lines tangent to the two orbits at the nodal points. In this approximation the averaged perturbing function has an analytical expression, allowing explicit computation with elliptic integrals and elementary functions.

There are two main applications of this generalized averaging. First, the Kozai librations of the argument of perihelion $\omega$, protected from node crossings, can be identified by means of the level lines of the averaged perturbing function: the libration centers correspond to minima of the averaged perturbing function, which can not occur on node crossing lines. There are also librations around maxima belonging to node crossing lines, with node crossings taking place in each cycle. Thus we have been able to prove (Gronchi and Milani, 1999) that some stable center of oscillation, either libration or circulation of $\omega$, always exists for every

given value of the integrals $a$ and $\sqrt{1-e^{2}} \cos I$, and this for an arbitrary number of perturbing planets. For objects which can cross the orbits of several planets, there are usually many such stable states, free from node crossings, with both symmetric and asymmetric librations.

Second, for both librations and circulations of the argument of perihelion $\omega$, it is possible to define proper frequencies for the angles $\Omega$ and $\varpi=\Omega+\omega$, and proper elements such as the maxima and/or minima of $e$ and $I$ over one cycle. To compute proper elements for given initial conditions we need to compute a solution of the generalized averaging equations, not only a level curve; this requires to find a numerical method to approximate the piecewise smooth solution of a differential equation with a singularity; at least one method exists (Milani and Gronchi, 1998), but its efficiency has yet to be investigated.

\section{References}

Gronchi, G.F., Milani, A.: 1998, 'Averaging on Earth-crossing orbits', Celest. Mech., in press.

Gronchi, G.F., Milani, A.: 1999, 'The stable Kozai state for asteroids and comets', Astron. and Astrophys.,, 341, 928-935.

Kozai, Y.: 1962, Astron.J., 67, 591-598

Milani, A., Gronchi, G.F.: 1998, 'Proper elements for Earth-crossers', IAU Colloquium 173, submitted. 